\title{
Enhancing the Performance of Fuel Cell Gas Diffusion Layers using Ordered Microstructural Design
}

DOI:

10.1149/2.0202001JES

Link to publication record in Manchester Research Explorer

\section{Citation for published version (APA):}

Niblett, D., Joekar-Niasar, V., \& Holmes, S. (2019). Enhancing the Performance of Fuel Cell Gas Diffusion Layers using Ordered Microstructural Design. Journal of the Electrochemical Society.

https://doi.org/10.1149/2.0202001JES

\section{Published in:}

Journal of the Electrochemical Society

\section{Citing this paper}

Please note that where the full-text provided on Manchester Research Explorer is the Author Accepted Manuscript or Proof version this may differ from the final Published version. If citing, it is advised that you check and use the publisher's definitive version.

\section{General rights}

Copyright and moral rights for the publications made accessible in the Research Explorer are retained by the authors and/or other copyright owners and it is a condition of accessing publications that users recognise and abide by the legal requirements associated with these rights.

\section{Takedown policy}

If you believe that this document breaches copyright please refer to the University of Manchester's Takedown Procedures [http://man.ac.uk/04Y6Bo] or contact uml.scholarlycommunications@manchester.ac.uk providing relevant details, so we can investigate your claim.

\section{OPEN ACCESS}


OPEN ACCESS

Enhancing the Performance of Fuel Cell Gas Diffusion Layers Using Ordered Microstructural Design

To cite this article: Daniel Niblett et al 2020 J. Electrochem. Soc. 167013520

View the article online for updates and enhancements. 


\title{
Enhancing the Performance of Fuel Cell Gas Diffusion Layers Using Ordered Microstructural Design
}

\author{
Daniel Niblett, 10 Vahid Niasar, $\mathbb{1}^{\mathrm{z}}$ and Stuart Holmes \\ Department of Chemical Engineering and Analytical Science, University of Manchester, Manchester, M13 9LP, \\ United Kingdom
}

\begin{abstract}
The Gas Diffusion Layer (GDL) is an important fibrous porous material within all fuel cells that manage the transport of electrons, heat and fluids in order to generate power. The microstructural morphology of electrically conductive solid porous media can be manipulated to produce structures with a larger effective electrical conductivity and reactant permeability when compared to current Gas Diffusion Layers (GDL) used in fuel cells. Using a numerical modelling approach, we simulated single phase flow and the electrical conductance in void and solid spaces, respectively. The simulations were completed in OpenFOAM which employs the finite volume approach. Simulations revealed that effective electrical conductivity is dependent on the electron path tortuosity $\tau_{E}$ and the porosity $\epsilon$. Therefore control of these micro-structural properties will allow for lower ohmic and mass transport losses. To aid the analysis, analytical and semi-empirical equations were developed based upon physical parameters to predict the effective electrical conductivity of connected porous media independent of isotropy. We propose that regular ordered structures via additive manufacturing techniques will allow for greater fuel cell performance.

(C) The Author(s) 2019. Published by ECS. This is an open access article distributed under the terms of the Creative Commons Attribution 4.0 License (CC BY, http://creativecommons.org/licenses/by/4.0/), which permits unrestricted reuse of the work in any medium, provided the original work is properly cited. [DOI: 10.1149/2.0202001JES]

(cc) BY
\end{abstract}

Manuscript submitted August 5, 2019; revised manuscript received September 30, 2019. Published November 19, 2019. This paper is part of the JES Focus Issue on Mathematical Modeling of Electrochemical Systems at Multiple Scales in Honor of Richard Alkire.

Fuel cells have the potential to provide an alternative to battery power electrical devices ranging from portable devices and automobiles. Their flexibility to be implemented along side battery powered vehicles to extend range, or coupled with electrolysers for enhanced use of renewable energy means that they are likely going to contribute to the society of the future. However, research into enhancing the performance of these devices continues and efforts are being made to analyze individual components of a fuel cell to understand the mechanisms behind performance loss. One such area is the Gas Diffusion Layer (GDL), which is a very significant component for managing heat, mass and electron transport. The structural properties of this layer are heterogeneous and spatially uncontrolled and consequently each transport involved within can be uncontrolled.

To reduce voltage losses caused by electrical resistance and flow resistance, the improvement of effective electrical conductivity and permeability can be considered. Conventional GDL materials are comprised of carbon paper; a web of micrometer sized carbon fibers (an example shown in (c) Figure 2), which can be considered to be an uncontrolled management of both electron and fluid transport either to or from the catalyst layer (CL). This uncontrolled transport can result in heterogeneous reaction rates under the rib and channel regions and ultimately causes a decrease in potential performance due to the flooding of the GDL with water; and therefore occupying pores for oxygen diffusion or resistance to electron flow.

The electrons flow through the solid carbon fiber material in the through-plane (TP) direction from the rib of the bi-polar plate to the $\mathrm{CL}$ as shown in Figure 1. Resulting from the manufacturing method used for generating GDL, the fibers are orientated in the in-plane (IP) direction, meaning electrons have a much less tortuous path in the IP direction which increases the effective electrical conductivity in that direction. ${ }^{1}$ The effective electrical conductivity tensor is important as it shows the anisotropy of the material, and as a result of this property, fibrous porous media do not correlate well with conventional correlations such as the Bruggeman's correlation since it is an empirical relation that has limited value outside porosity values above $0.7{ }^{2}$ Furthermore, it does not consider the 3D microstructure topology. ${ }^{3}$

The reactants flow through the void space generated by the 3D microstructure and therefore due to the random nature of fibrous materials, will generate a flow path that will be not optimized for transporting the reactants to the CL and removing the products from the GDL via the gas channels. In most fuel cells at the cathode, there is two-phase

${ }^{\text {z} E-m a i l: ~ v a h i d . n i a s a r @ m a n c h e s t e r . a c . u k ~}$ flow of air (reactant) and water (product), shown in Figure 1, which have interactions with the microstructure and each other due to the different wettability surfaces. Currently, fuel cells have problems with water flooding at high current density and as of yet there has been little study to improve the structure to attempt to address this challenge, instead PTFE coating is often used which renders the fibers more hydrophobic. The distribution of the PFTE may be not favorable and can create zones of heterogeneous wettability. Attempts to improve the arrangement of PFTE on a GDL has been conducted with significant improvements to performance. ${ }^{4}$

In this study, numerical simulations were used to evaluate the performance of ordered micro-structures shown in Figures $2 a$ and $2 b$ in comparison to the conventional GDL. We also showed that the lattice structure can be manufactured using appropriate additive manufacturing techniques as shown in Figure 3. Furthermore, resulting from the emergence of cheaper and higher resolution additive manufacturing methods, ${ }^{5}$ it is possible that these structures can be made in the present day shown by our recent sample produced in kind by Microlight 3D based on one of our lattice designs shown in Figure 3, assuming the structures can be carbonized to the same material as that of conventional GDL (carbon fiber). In this paper we simulate the pore-scale single phase flow in the void space and electron transport in the solid

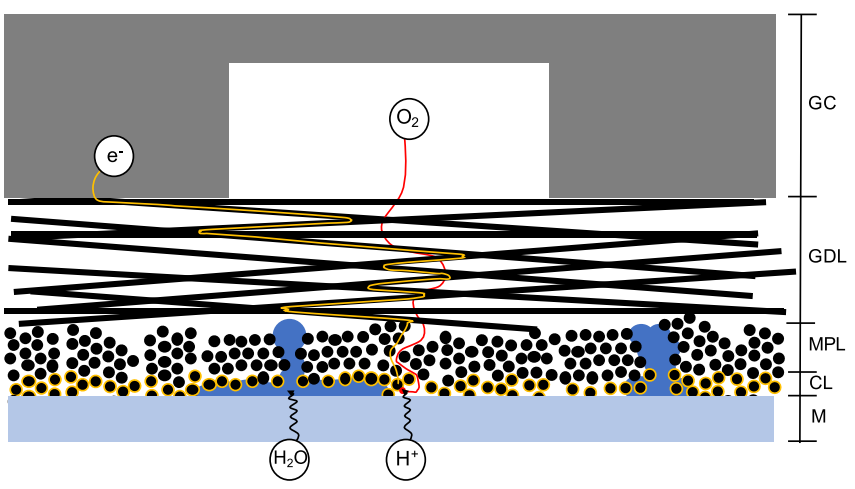

Figure 1. Schematic of the components within a fuel cell, illustrating the dual transport mechanisms of the GDL. Electrons are transported in the solid fibers (yellow), while reactants (oxygen) and products (water) are transported through the void space (red). Gas Channel (GC), Gas Diffusion Layer (GDL), Microporous Layer (MPL), Catalyst Layer (CL) and Membrane (M) are shown on the diagram. 


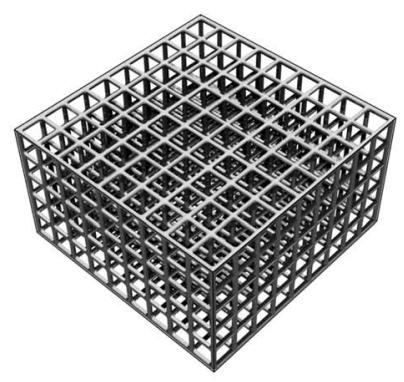

A

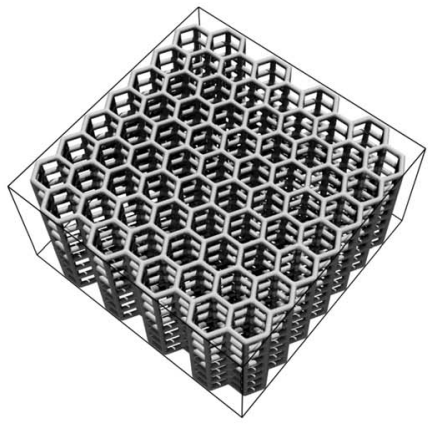

B

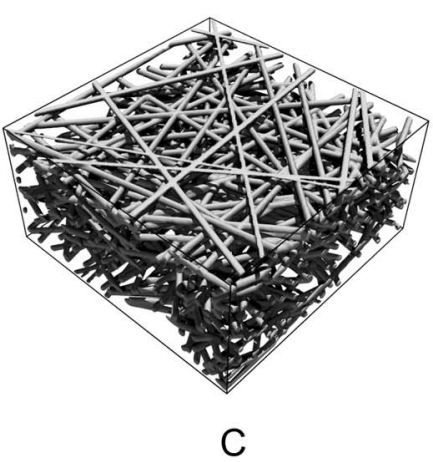

C

Figure 2. (A), (B) and (C) show the microstructures generated and tested within this study. The cubic lattice (isotropic) (A), hex lattice (anisotropic) (B) and GDL (carbon paper reconstruction) (C). All of the structures have a thickness of $200 \mu \mathrm{m}$ and a fiber diameter of $9 \mu \mathrm{m}$.

space in structured and unstructured GDL and compare the flow and transport properties with the conventional GDL.

Although two-phase simulation is outside of the scope of this study, increasing the single phase permeability to be larger than that of conventional GDL; which are chosen because they have a high porosity and therefore permeability, has the potential to alleviate mass transport losses. This is because the diffusive resistance will additionally be reduced as the fluid phase pathways will be less tortuous. Although the two-phase flow properties are vastly more important at higher current density, at low current density, it is possible that the fuel cell can operate with vapor diffusion only.

\section{Literature Review}

Looking at the properties of an ideal GDL, there are a variety of functions that should be satisfied by conventional carbon paper or cloth materials such as:

- allowing path for electron conduction between biploar plates and catalyst layers,

- promoting reactant and product transport to and from the catalyst layers,

- transferring heat from the CL to the bipolar plate,

- providing mechanical support to the membrane. ${ }^{6}$

Considering these performance criteria, it is apparent that conventional GDL materials have not been optimized for these purposes. This is clear from the apparent spatial heterogeneity and thin porous microstructure that they are comprised of Ref. 7. Optimisation of microstructural morphology could appropriately enhance each of the criteria listed above, providing an improvement over conventional GDL through regular ordered microstructures.

Improvement to fuel cell performance by changing the GDL properties has been simulated in a variety of studies which show the effect of varying the effective electrical conductivity, ${ }^{8-10}$ permeability, ${ }^{10}$ effective thermal conductivity ${ }^{11}$ and isotropy of the GDL. ${ }^{8,12}$ However, to our knowledge there is no study that has proposed to change the mor- phology of the GDL to enhance flow properties of fluids and transport properties for the electrons.

The impact of increasing the effective electrical conductivity in the TP direction on fuel cell performance has been conducted through simulations ${ }^{9}$ where the IP electrical conductivity was increased from 500 to $10000 \mathrm{~S} \mathrm{~m}^{-1}$ and the resulting current density was increased from $50 \mathrm{~mA} \mathrm{~cm}^{-2}$ to above $200 \mathrm{~mA} \mathrm{~cm}^{-2}$ at an operating voltage of $0.85 \mathrm{~V}$. Therefore this provides evidence that increasing the electrical transport properties of the GDL will improve the fuel cell performance. However, the effect of increased water production on the relative permeability of the GDL and consequently reducing the reaction rate due to lower oxygen concentration at the CL were not considered.

Simulation of fuel cell performance using a 1D model has been used $^{8}$ to show that the voltage loss due to ohmic loss can be reduced by using an electrode with higher electrical conductivity. Additionally the amount of voltage loss can be quantified using a simple equation that contains resistances from each component; allowing the contribution of the GDL resistance to be compared against the rest of the components. Further studies ${ }^{10}$ take this further, which involves numerical simulations to evaluate the effect of permeability and electrical conductivity on the performance on fuel cells. They found that the increasing the electrical conductivity of the TP direction from $48 \mathrm{~S}$ $\mathrm{m}^{-1}$ to $4000 \mathrm{~S} \mathrm{~m}^{-1}$ (which is a common value for the IP conductivity) increases the current density because the activation over potential will scale. Therefore, if a structure can be made with higher conductivities, improved performance can be expected.

A different model which aims to show the effect of varying the isotropy of the GDL on the performance of a fuel cell ${ }^{11}$ only evaluated the change of thermal conductivity through isotropy, whereas if the GDL was isotropic, the effective electrical conductivity and the permeability will vary which will effect the performance differently to what is reported. Furthermore other studies ${ }^{12}$ using a fuel cell simulation, report using a more isotropic GDL has an improvement on fuel cell performance; although they state that the performance is overpredicted for their isotropic case.

Complete fuel cell modelling studies involving connected electrically conductive porous such as GDL, media may benefit from a

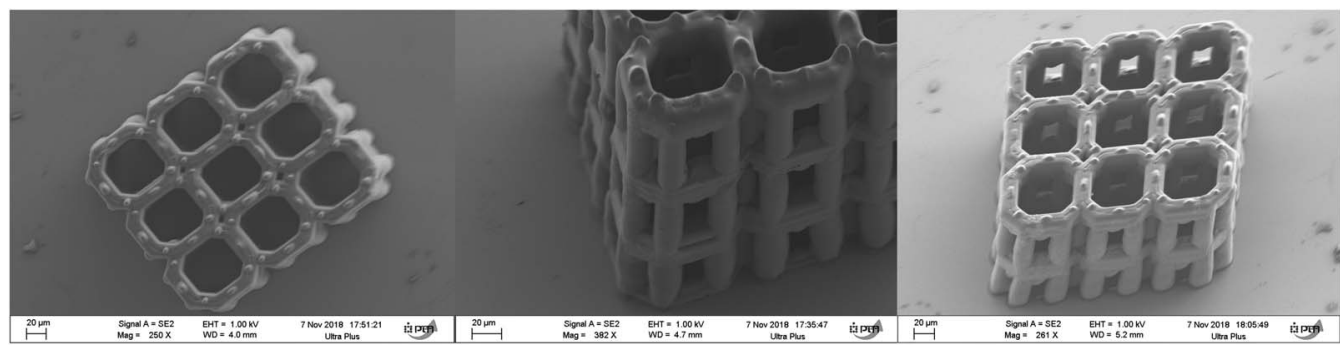

Figure 3. Microscope images of additive manufactured samples of an ordered lattice structure with fiber diameters of $20 \mu \mathrm{m}$ and domain size of $300 \mu \mathrm{m}$ produced by high resolution two-photon polymerisation technique. These resolution of these structures after carbonisation will be similar to that of traditional carbon paper. 
expression that can predict effective electrical conductivity, accurately, independent of isotropy. The use of a analytical expressions which include the 3D microstructure for the determination of effective transport properties are useful in parameterisation of GDL. The need of understanding morphology has been highlighted in the literature such as the well-known Bruggemann's correction to determine the transport properties. ${ }^{13}$ Furthermore, similar studies ${ }^{14}$ employ the use of such effective transport parameters to determine the performance of PEMFC operating at high current density. However, they require separate equations for the in-plane and through-plane electrical conductivity which is only a function of porosity. Our studies show that there is another parameter that must be considered for predicting the electrical conductivity of connected porous media. The work involved within this study aims to develop a more detailed expression for predicting effective electrical conductivity for all microstructures independent of isotropy; which is performed by microstructural morphology simulations.

Numerical simulations on fibrous $\mathrm{GDL}^{3}$ provide a correction to the Bruggeman's correlation and show the clear variation between IP and TP electrical conductivity, however mechanistically the expression can be reduced further by introducing another variable $\tau_{e}$ that is common in all connected electron transport media.

Instead of using a different variation of GDL to improve fuel cell performance, this study proposes a novel concept of implementing a ordered micro-structure to improve performance for both fluid flow and electrical conductance. Improving GDL permeability has the potential to allow higher current densities to be achieved because the concentration of reactant at the reaction zones will be larger, resulting in a fast rate of reaction. ${ }^{15}$ Conventional GDL are highly anisotropic, meaning that the in-plane conductivity is usually one order of magnitude higher than the through-plane conductivity; ${ }^{1}$ an isotropic ordered structure can be greater than both.

A 3D printing technique available to manufacture such a structure that is able to be placed inside a fuel cell is Two-Photon Polymerisation (TPP). Polymeric microstructures can be fabricated by scanning the laser beam inside a photopolymer layer deposited on a substrate. This technique is able to produce complex microstructures like microlattices with resolutions of $1 \mu \mathrm{m}$. The application of 3D printing to fuel cell gas diffusion layers can be accomplished by using a method in Ref. 5 where new photopolymers are tested for shrinkage ratio and mass loss after carbonisation processes. The structures that were manufactured had an electrical conductivity between $529 \mathrm{~S} \mathrm{~m}^{-1}$ and $769 \mathrm{~S} \mathrm{~m}^{-1}$ which are in the range of conductivity that would make the material applicable for replacing GDL in fuel cell applications. Furthermore, if optimization of the technique or material is improved, higher conductivities could be expected if the carbonisation process follows a similar method to that used in common carbon fiber manufacturing process; utilizing three stages of heating to improve the quality of carbon structures. This should be possible as presented in studies where electrical conductivities of $10000 \mathrm{~S} \mathrm{~m}^{-1}$ can be reached at a temperature of $1000^{\circ} \mathrm{C} .{ }^{16}$ However as shown by both studies, the effects of high temperature treatment may affect the mechanical integrity of structures developed.

The integration of a 3D printed GDL into electrochemical devices has only been performed by two studies as far as is known. ${ }^{17,18}$ In an electrolyser (where the two-phase flow processes are similar to that of an anode side direct methanol fuel cell) a 3D printed gas diffusion layer has been tested. ${ }^{17}$ The ohmic loss was significantly decreased from $0.47 \Omega \mathrm{m}^{-1}$ to $0.36 \Omega \mathrm{m}^{-1}$ which was a consequence of the change in interfacial contact resistance. However although they did not test it, the change in morphology to a regular structure will improve electrical conductivity. In these devices, regular carbon paper or cloth cannot be used due to the high electrical conductivity needed; it is possible that the structures developed in this study may be able to be implemented in electrolysers due to the improved electrical conductivity. Effective 3D printed GDL's will promote a uniform current and thermal distribution at the adjacent reaction sites meaning there is not heterogeneous reaction activity in different regions. The use of a low-cost 3D printing method to develop structured titanium gas diffu- sion media resulted in well controlled two-phase transport ${ }^{17}$ and high electrical and thermal conductivity. Their study generated different liquid GDL square mesh which had thicknesses of around $500 \mu \mathrm{m}$. This study provides confidence behind a structured GDL, especially one that considers two-phase flow and electron transport.

Furthermore, a 3D printed GDL was used in different a different study ${ }^{18}$ which explains that a $3 \mathrm{D}$ printed manufacturing technique can be more cost effective than manufacturing a regular GDL and uses a polyamide/Titanium structure to generate a GDL. Their method of 3D printing did not control the microstructural morphology, but created a different uncontrolled structure with a different chemical structure. The resulting performance is far lower than the conventional GDL material and this is due to the low electrical conductivity; induced by uncontrolled porous media morphology and potentially lower transport control. The transport properties were not considered in this study and therefore the performance was lower.

The design choice for testing a cubic lattice is derived from the investigations of maximum permeability structures from Refs. 1921 where the optimization of two dimensional and three dimensional isotropic permeability results in a solid void structure that resembles that of a unit cell for a cubic lattice. This is because the void space of a cubic lattice fibrous structure has the lowest surface area for a given porosity and resembles a P-Schwarz triply periodic minimal surface which structure can be seen in Ref. 20. This minimal fluidsolid interaction would reduce resistance because there is less contact with the walls where the velocity is zero. The optimization of electrical and thermal conductance was carried out ${ }^{19}$ which resulted in the same type of structure being generated. However, in this study, the purpose is to optimize electrical conductivity and permeability in terms of fuel cell performance, therefore the performance characteristics of fuel cells must be considered in the choice, including the limit of the bulk material it can be made from.

There is spatial inhomogeneity in the porosity of thin fibrous porous media and so in some locations the material may have higher transport properties than in other areas. ${ }^{7}$ Whereas the effective diffusivity is important for oxygen diffusion, and models include the effect of the two-phase flow ${ }^{22}$ on this parameter, in this study we are only looking into the effect of the micro-structure on the electrical and fluid permeability. The type of modelling using relative permeability and capillary pressure saturation curves may not be applicable for these types of thin porous media especially when dealing with distinct independent injection sites to the GDL.

Simulation studies into the analysis of the representative elementary volume (REV) and through plane regional characteristics on the effective transport properties of carbon fiber GDL has shown that an REV cannot be defined due to the separation between a pore and volume averaged scales ${ }^{7}$ and so its properties can change vastly over the material. While this is acknowledged, the work presented in this study proposes an idealized case related to the microstructure alone.

\section{Methodology}

Optimisation of the GDL required development and creation of geometry for a regular carbon paper GDL; as a benchmark for both the solver and to compare the performance of regular lattice structures. These structures were generated and manipulated using AutoDesk AutoCAD, MATLAB and Elements by nTopology. The three dimensional meshing was performed using the native meshing tool of OpenFOAM; snappyHexMesh. The following areas were developed in order to simulate and evaluate each case:

- Computational domain generation with carbon paper reconstruction and ordered lattice generation

- Development of solver for OpenFOAM

- Development of analytical solution for effective electrical conductivity for deeper understanding.

Numerical domain development.-Unstructured - gas diffusion layer generation.-The method used to generate numerical 

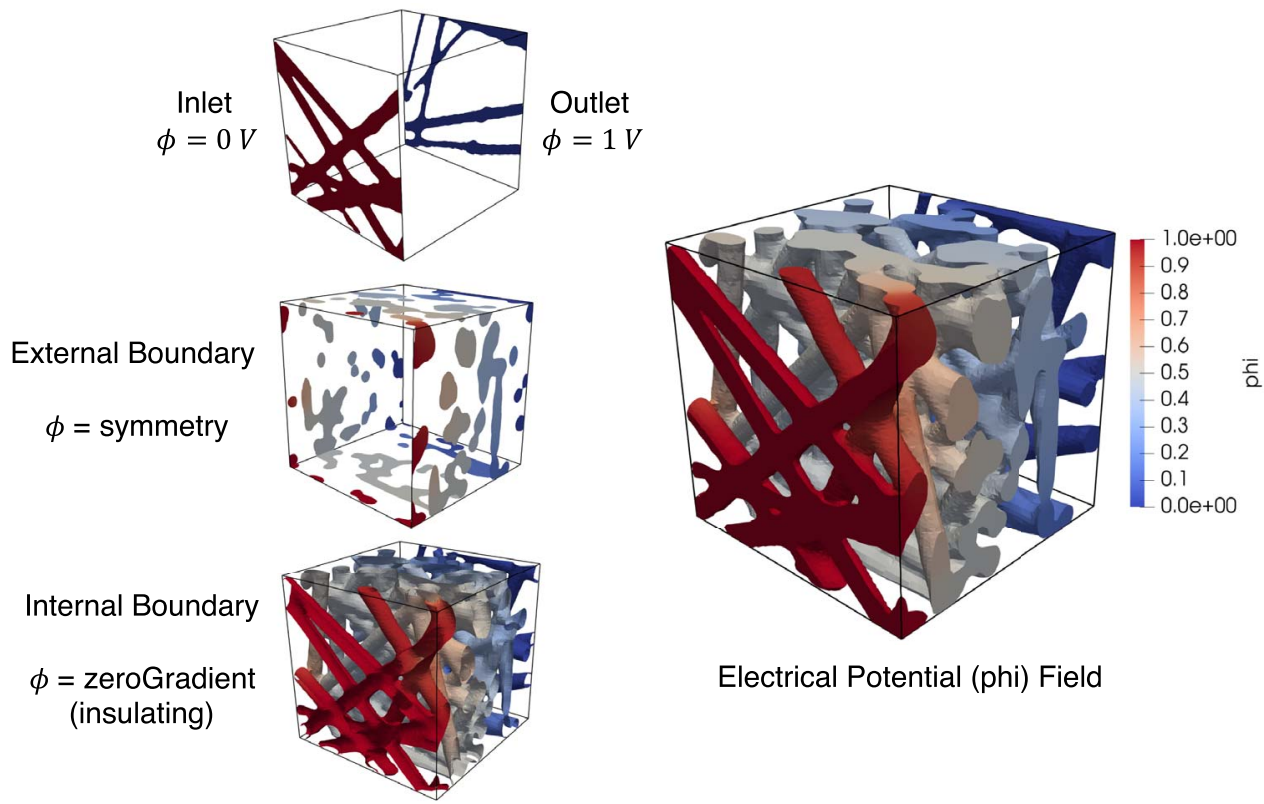

Electrical Potential (phi) Field

Figure 4. Boundary conditions for the eletrical current simulations, including the simulated electrical potential field on the right.

reconstruction of the GDL topology is a stochastic method taken from Ref. 23. Its use has been supported in literature as shown by reviews on microstructural design of GDL by Refs. 6,24,25 where stochastic methods are one of the most common methods of generating reconstructions of GDL. In our study, cubic numerical domains with side lengths of between $100 \mu \mathrm{m}$ and $300 \mu \mathrm{m}$ were used for the simulations. An example of a $100 \mu \mathrm{m}$ domain is shown in Figure 4 . The reliability of using this scale of domain is shown by the small variation in porosity and permeability as the size is changed. Furthermore, other simulation studies use similar numerical domain sizes such as ${ }^{1}$ which used a domain size with side lengths of $200 \mu \mathrm{m} \times 200 \mu \mathrm{m} \times 170 \mu \mathrm{m}$. The study by Ref. 3 quantifies the homogeneous coefficient of variation between different GDL domain sizes on effective electrical conductivity in cubic domains ranging from 50 to $400 \mu \mathrm{m}$. Their study showed that within the range of cubic domains, coefficient of variation for each porosity is low. Both of these simulation results are validated against experimental data, giving confidence that the GDL structures developed in this study are representative for realistic GDL.

Experimental and numerical simulation results for GDL permeability and conductivity have previously been evaluated in literature ${ }^{23}$ which used a similar method to construct and simulate the GDL as used in this study. They clearly show the difference between the IP and TP electrical conductivity and permeability. The results are useful for the determination of the validity of the numerical solver we present here. Furthermore the experimental results by Ref. 18 are useful for visualizing the range of GDL transport properties for the in-plane direction so that they can be used for comparison against our proposed GDL structures.

An accurate reconstruction of carbon paper GDL that is used within fuel cells should contain: the carbon fibers, carbonaceous binder and a PTFE coating. However, since this study is about the base structure, only the carbon fibers are included but as shown by Ref. 25 the addition of binder or PTFE does not vary the electrical conductivity significantly since electrons prefer to travel through the highly conductive carbon fibers. Experimental results by Ref. 10 show that the electrical conductivity of PTFE treated GDL in the IP direction is almost constant and therefore the decision in this study to neglect the PTFE coating is valid.

Since the focus of this paper is the impact of the topology and geometry on GDL performance, variation in the effective electrical conductivity has not been considered. Development of reconstructed GDL microstructures at different porosity; that is connected, is used for computational simulations through finite volume methods. Consequently, the following assumptions are adopted using the method by Ref. 23:

1. Fibres are constructed from infinitely long circular cylinders made from graphite (assumed bulk conductivity $\sigma=100,000 \mathrm{~S} \mathrm{~m}^{-110}$ ).

2. Fibres are allowed to overlap, intersections are neglected; to allow for electron flow continuity.

3. The structure is highly anisotropic, with fibers aligned in the xy plane. The distribution properties for stochastic generation are invariant with respect to translations as well as rotations in the z-axis.

4. Side length of the computational domain was limited to $300 \mu \mathrm{m}$ with the same cell size but with a different total number of cells per simulation. Thickness of GDL used in commercial fuel cells are usually around $200 \mu \mathrm{m} .{ }^{26}$ Generated domain was larger than this, shown in Figure 2.

5. Fiber diameters generated $(9 \mu \mathrm{m})$ are within the range of commercial GDL materials used in real fuel cells. ${ }^{27}$

The assumptions stated above satisfy a stationary Poisson line process with a one-parametric directional distribution; cylinders are attached to the center of each line. The directional distribution is given in polar coordinates by its density and therefore $p(\theta, \Phi)$ is a function of altitude and longitude.

$$
p(\theta, \Phi)=\frac{1}{4 \pi} \frac{\beta \sin \theta}{\left(1+\left(\beta^{2}-1\right) \cos ^{2} \theta\right)^{\frac{3}{2}}}
$$

where $\beta$ is the anisotropy parameter ${ }^{23}$ which when $\beta=1$ an isotropic system is described and $\beta=\infty$ the system becomes more parallel to the X-Y plane. For the regular GDL structures, using a value of $\beta=10000$ is in line with others in literature ${ }^{3,23}$ since using a large value will suppress the fibers orientated in the $\mathrm{z}$-direction which more closely resembles the fibers generated by the traditional Electrospinning process.

GDL reconstructions with $\beta=10000$ were generated for variety of porosity (0.5-0.9), cubic domain sizes (with side lengths of 100 , 200 and $300 \mu \mathrm{m})$ and fiber diameters $(7,9 \mu \mathrm{m})$ which are similar to those reported for commercial GDL. ${ }^{15}$ These structures were used to represent the real GDL structures to obtain the simulation results in Figures 6 and 7. Furthermore, to test how anisotropy affects the 
electrical conduction shown in Figure 8, artificial GDL samples with $\beta=1-40$ were generated at constant porosity $(0.6,0.7$ and 0.8$)$.

Structured - cubic and hexagonal lattice generation.-Changing the morphology of the GDL reconstructions to create equivalent lattice structure requires solid phase continuity to be conserved which is achieved by using the same cubic domain size (with side lengths of $100-300 \mu \mathrm{m})$, porosity $(0.6-0.9)$ and fiber diameter $(9 \mu \mathrm{m})$ as the GDL reconstructions. This volume conservation of the solid phase was maintained at each lattice structure created, however to look at the variation of effective electrical conductivity and permeability with domain size, the cubic lattices were tested at a variety of scales as well as with the GDL reconstructions.

The cubic and hexagonal lattice represent an isotropic and anisotropic microstructures respectively. This allows for evaluation of the structural property importance on variation of the TP and IP transport of both electrons and fluids. With both lattices, the number of internal fibers that are introduced will increase the porosity, affecting the permeability, but should only slightly affect the electron conductivity (due to tortuosity induced at connecting nodes visualized in the simulations by the streamlines in Figure 12). The hexagonal and cubic lattice structures were generated using the software Elements Free by nTopology and MATLAB. Visualisation of the microstructures tested can be seen in Figure 2.

Governing equations.-To determine the effective electrical conductivity and the permeability of the micro-structures the electrical and pressure potential must be solved in the solid and void spaces, respectively. The study involves the use of finite volume methods which follows that of similar use in literature for similar studies. ${ }^{3,23}$ The use of this method allows for the realization of the effect of microstructural morphology on the transport properties. The structures are generated using MATLAB surface generation and STL extraction and then further meshed using the native meshing tool snappyHexMesh in OpenFOAM. The simulations were performed using different solvers of OpenFOAM.

Effective electrical conductivity.-The effective electrical conductivity tensor $\sigma^{e f f}$ is determined by the specification of electrical potential $\phi$ at opposite sides of the domain as shown in Figure 4. The diagonal elements in the tensor are the resulting effective electrical conductivity for the $\mathrm{x}, \mathrm{y}$ (in-plane) and $\mathrm{z}$ directions (through-plane). A solver made from the adaptation of the laplacianFoam solver in OpenFOAM to solve for electrical potential and current. Using Ohm's second law, the conservation of electron transport is applied to the solid region; electrons do not flow through the void space.

$$
\nabla \cdot(\sigma \nabla \phi)=0
$$

where $\phi$ is the electrical potential $(\mathrm{V})$ resulting from the flow of electrons to or from the CL. $\sigma$ is the bulk electrical conductivity of the solid material $\left(100,000 \mathrm{~S} \mathrm{~m}^{-1}\right)$ and therefore the current can be determined by

$$
i=\sigma \nabla \phi .
$$

The boundary conditions for the domain (see Figure 4) is specified so that there is no electrical conduction between the solid fibers and the void space and that the structure is mirrored on the external boundaries. A voltage of $0 \mathrm{~V}$ is specified at the inlet and $1 \mathrm{~V}$ is specified at the outlet, symmetry boundary conditions are applied on the lateral walls which mimics a continuation of the structure in the $\mathrm{x}$ and $\mathrm{y}$ directions. Solving the three auxiliary equations in each direction will allow the average current density to be calculated.

$$
i=\frac{1}{A} \int-\sigma(n \cdot \nabla) \phi d A
$$

where $i$ is the average current density $\left(\mathrm{A} \mathrm{m}^{-2}\right)$ which is evaluated over the solid cross sectional surface area in the direction normal to the specified inlet and outlets. The average current density is evaluated at the inlet and outlet cross section to ensure there is no significant error in current. The effective electrical conductivity can be determined from the theory of resistors

$$
R=\rho \frac{L}{A}
$$

where $R$ is resistance, $\rho$ is resistivity $\left(\frac{1}{\sigma}\right), L$ is the length of the conductor and $A$ is the cross sectional area for electron flow. Using Ohm's law $(V=I R)$ the effective electrical conductivity can be determined as

$$
\sigma^{e f f}=\frac{I L}{A \Delta \phi}
$$

which in the simulations, $L$ is the distance between the inlet and outlet and $i=\frac{I}{A}$ therefore, the conductivity is finally determined through

$$
\sigma^{e f f}=\frac{i L}{\Delta \phi}
$$

The simulations were run with a tolerance of $10^{-10}$ for electrical potential and current which will enforce the electron conduction continuity. Additionally, the average current at 3 cross sections (inlet, outlet and middle section) was evaluated to validate continuity.

Permeability simulation.-The permeability was determined through the microscale single phase simulation of the flow of water through the void space defined by the microstructural geometry. This is the opposite to the solid void where the electrons flow. Here it is considered that the flow is non-inertial single phase flow characterized by low Reynolds numbers that allows the use of Stokes' equations. ${ }^{28}$ At the scales experienced of flow through GDL, gravity can be neglected and at steady state, the conservation of mass and momentum results in:

$$
\begin{gathered}
\nabla \cdot u=0 \\
\mu \Delta u=\nabla p
\end{gathered}
$$

Where $u$ is the velocity and $p$ is the pressure. Equation 9 can be reformulated at the macroscale into Darcy's Law

$$
v=-\frac{K}{\mu} \cdot \nabla P
$$

$v$ is the Darcy velocity and $K$ is the permeability tensor defined by the direction of the velocity and the pressure gradient. The velocities are integrated over the total volume of the void space

$$
v_{i}=\frac{1}{V} \int u(i) d V
$$

where $v_{i}$ is the Darcy velocity in the $\mathrm{x}, \mathrm{y}$ or $\mathrm{z}$ direction. This was then used to determine the diagonal elements of the permeability tensor of the structure by

$$
K_{i j}=-\frac{v_{i} \mu}{\Delta P_{j}}
$$

The boundary conditions applied to the numerical domain (fluid space) is shown in Figure 5.

Computational mesh analysis.- The computational mesh was developed using the meshing tool of the open-source CFD toolbox OpenFOAM, snappyHexMesh. A hexahedral mesh was generated to match the surface of a given geometry. To evaluate the solution dependence on the mesh resolution, 4 different cases were simulated on a cubic domain with side lengths of $100 \mu \mathrm{m}$ with a carbon paper reconstruction with different cell resolutions $(3,2,1,0.8 \mu \mathrm{m})$ and the error in the effective electrical conductivity and the permeability was determined. The error in the effective electrical conductivity between $2 \mu \mathrm{m}$ and 0.8 $\mu \mathrm{m}$ was less than $1 \%$. The difference in permeability between 2 and $0.8 \mu \mathrm{m}$ was less than $5 \%$. From this analysis, a cell resolution of $1 \mu \mathrm{m}$ ( $260 \mathrm{~K}$ cells for cubic domain with side lengths of $100 \mu \mathrm{m}$ ) and $2 \mu \mathrm{m}$ ( $126 \mathrm{~K}$ cells for cubic domain with side lengths of $100 \mu \mathrm{m}$ ) was used for the electron transport and single phase flow solvers respectively. This resulted in the largest simulations for single-phase flow being 


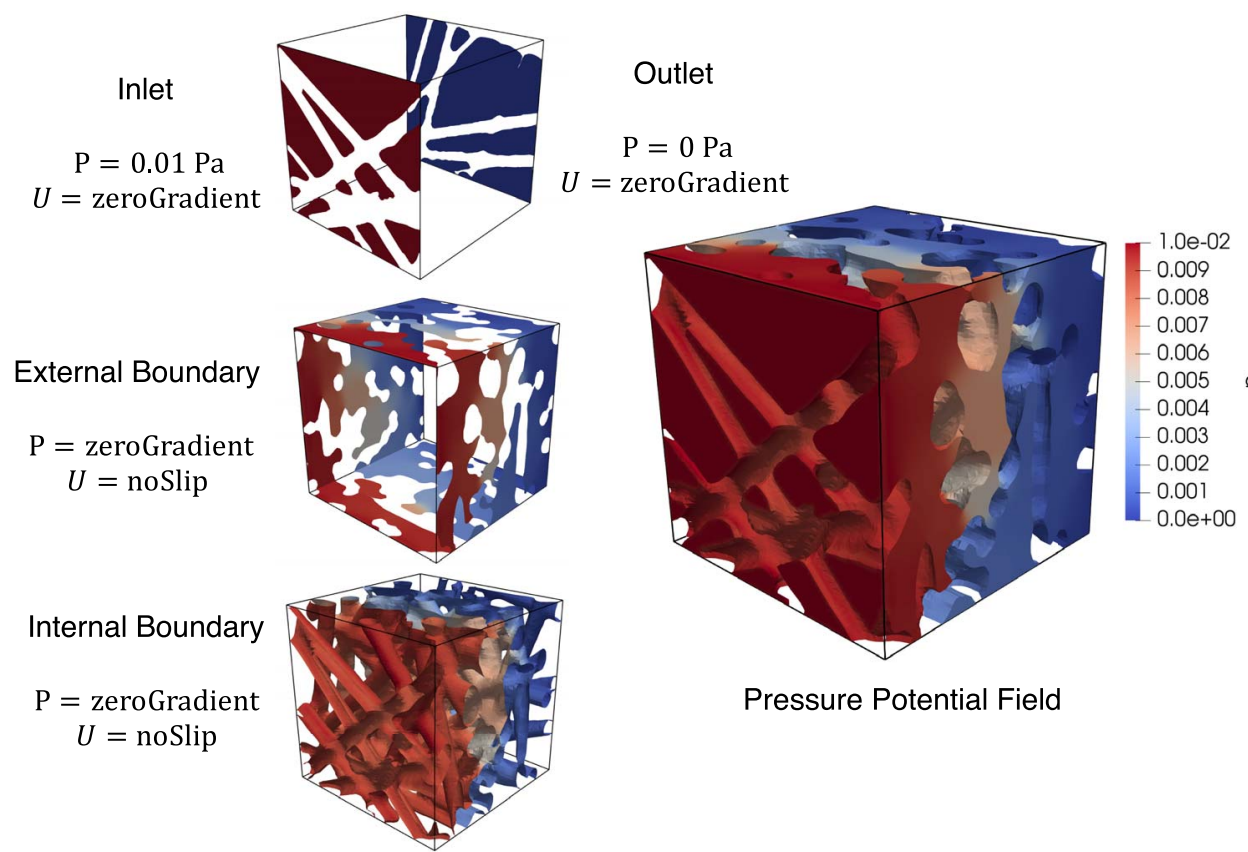

Figure 5. Boundary conditions for the single phase flow simulations, including the simulated pressure field on the right.

comprised of over 3.4 million cells. All simulations had a tolerance of $10^{-10}$ for velocity and pressure. The solver is based upon the SemiImplicit Method for Pressure Linked Equations (SIMPLE) algorithm and the discretization schemes used are second order.

Effective electrical conductivity analytical solution for cubic lattice.-To further validate the simulation results and to understand the process of how micro-structural morphology affects the electrical conductivity an analytical model was developed. The simulation results showed that lattice fibers parallel to the electric potential did not conduct electron flow and therefore the effective area that electrons flow through is not the cross sectional area of the structure. Therefore for a cubic lattice the cross sectional area for electron flow can be

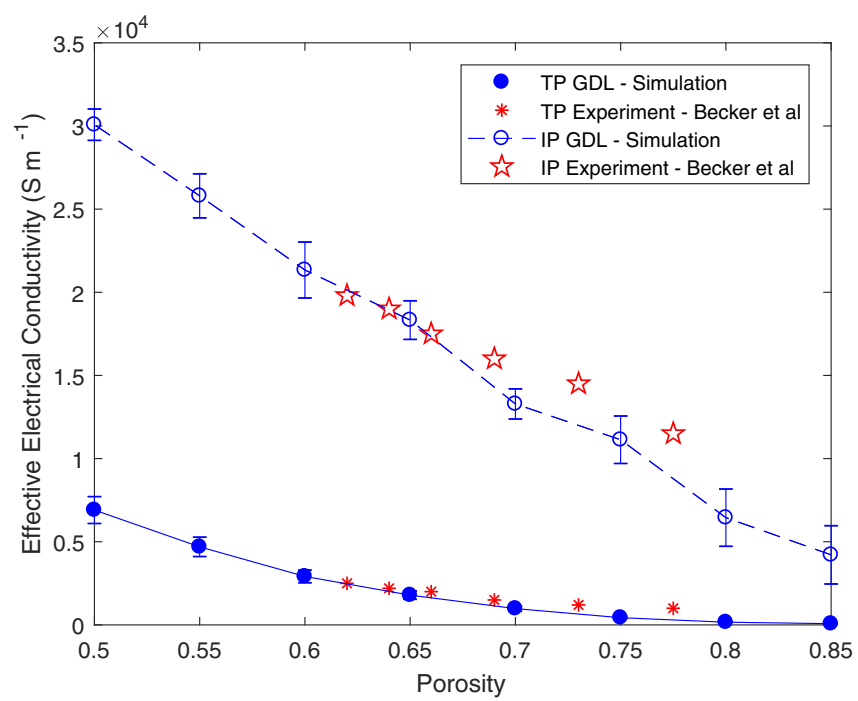

Figure 6. Results of the Through-Plane (TP) and the In-Plane (IP) effective electrical conductivity simulations for GDL micro-structures generated at different porosity. The red points represent the experimental values taken from literature ${ }^{27}$ and the solid points represent the TP direction. The error bars are the standard deviation between the different domain size GDL structures generated (100 to $300 \mu \mathrm{m}$ ) and simulated. considered to be a bundle of cylindrical fibers. Resistance and current of each fiber is known by Equation 5 and Ohms law

$$
\Delta V=I R
$$

The number of tubes $N$ for a cubic lattice that electrons pass through with $U_{C}$ cells is

$$
N=\left(U_{c}+1\right)^{2}
$$

The total current can be therefore determined by considering the use of Equations 5, 13, 14 to the system shown in Figure 4

$$
I=\frac{A_{t} N \Delta V}{\rho L_{t}}
$$

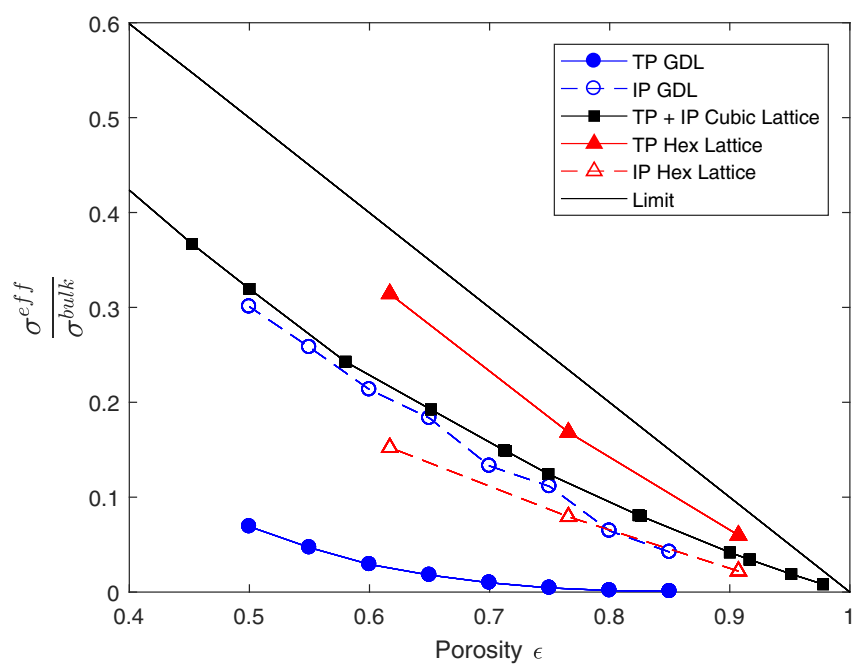

Figure 7. Comparison between GDL, cubic lattice and hex lattice structures in the TP and IP direction. The TP direction are solid whilst the IP direction is hollow. GDL is represented in blue, cubic lattice in black squares, and hex lattice as red triangles. The black solid line represents the limit of conductivity achievable which was found by the simulation of a bundle of isolated fibers. 


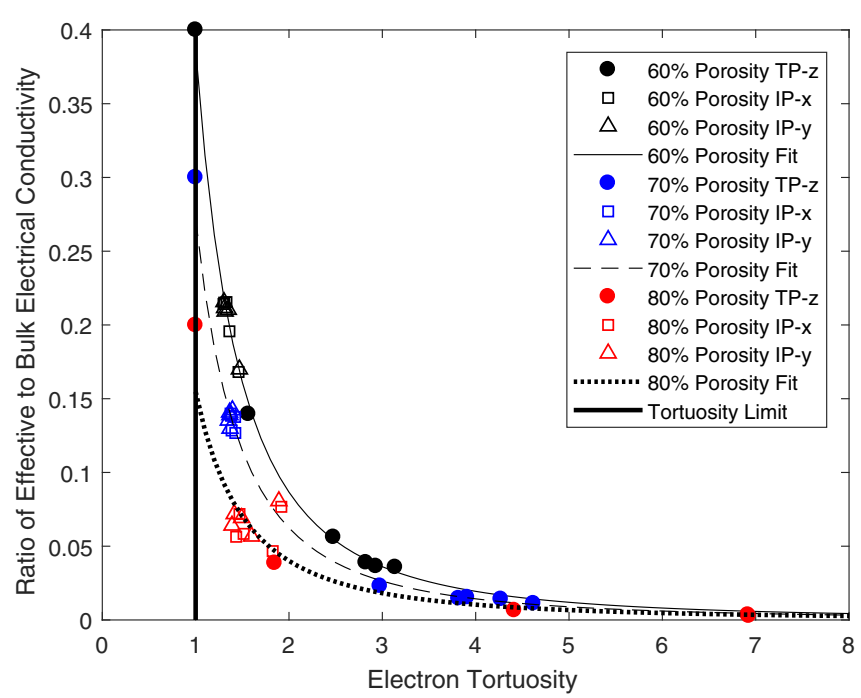

Figure 8. Comparison of the ratio of effective to bulk electrical conductivity and electron tortuosity between different anisotropy GDL structures of a different porosity $(0.6=\mathrm{red}, 0.7=$ blue, $0.8=$ black $)$. The solid circles are the TP direction, hollow squares are the IP-x direction and hollow triangles are the IP-y direction. The Thick solid black line represents the limit of tortuosity which is 1 and the points that lie on this line represent the simulation of a bundle of fibers that are completely aligned with the $\mathrm{Z}$ axis and therefore have no tortuosity.

For a specified domain length of $L_{D}$ and an area of $A_{D}$ effective electrical conductivity can be determined using the total current $I$ from Equation 15

$$
\sigma^{e f f}=\frac{I L_{D}}{A_{D} \Delta V}
$$

The combination of Equations 15 and 16 will allow an analytical equation to be developed based upon physical properties of the lattice microstructure. The effective electrical conductivity is therefore

$$
\sigma^{e f f}=\frac{A_{t} N L_{D}}{A_{D} \rho L_{t}}
$$

Terms within Equation 17 can be simplified into common expressions for porous conductive microstructures such as electron tortuosity and effective porosity, where electron tortuosity is defined as

$$
\tau_{e}=\frac{L_{t}}{L_{D}}
$$

$L_{t}$ is the length of the tortuous electron stream tubes, and $L_{D}$ is the length of the domain from the inlet to the outlet electrical potential. Additionally, the effective porosity is the $2 \mathrm{D}$ cross sectional porosity of the domain and can be defined from the area of a fiber $A_{t}$, the number of fibers $N$ and the domain area $A_{D}$.

$$
\epsilon^{e f f}=1-\frac{A_{t} N}{A_{D}}
$$

The rearrangement of Equations 19 and 18 allows Equation 17 to be simplified into Equation 20 that relates the effective electrical conductivity to only the effective porosity, bulk resistivity and electron tortuosity

$$
\sigma^{e f f}=\frac{\left(1-\epsilon^{e f f}\right)}{\rho \tau_{e}}
$$

These parameters are purely geometrical meaning that is is possible to see that the if the electron tortuosity increases, the effective electrical conductivity will decrease. Furthermore, the effective porosity will be different than the $3 \mathrm{D}$ porosity and should be the actual area that electrons pass through. The validity of Equation 20 will be evaluated against simulated cubic lattice structures. To compare only microstructure effects, regardless of bulk material they are comprised of, the dimensionless ratio of effective to bulk electrical conductivity is defined in Equation 21.

$$
\sigma=\frac{\sigma_{e f f}}{\sigma_{b u l k}}
$$

\section{Results}

Electrical conductivity.-The simulation of the electron conduction through the solid microstructures allowed the paths for electron flow to be evaluated. The electrons flow down the path of least resistance and therefore for instances such as in a GDL, the electrons can travel much more easily in the IP direction compared to the TP direction, because the fibers are aligned in the IP direction.

Figure 6 gives confidence that the solver can determine the effective electrical conductivity reasonably well for both the TP and the IP direction. Furthermore, the results are similar to those in literature. ${ }^{3,27}$ The assumptions used in the reconstruction of the GDL resulted in fibers that were allowed to intersect, whereas fibers realistically overlap and often have a carbonaceous binder that allows for facilitation of electron transfer. The reason for the variability of the structures (represented by the standard deviation for each micro-structure) is because the morphology of each domain is different, meaning there will be a variation in the electron tortuosity. The IP electrical conductivity is 1 order of magnitude larger than the TP, and the effect of this means that in a real fuel cell application, electrons are distributed heterogeneously across the layer. The electrical conductivity can be non-dimensionalized by dividing the effective electrical conductivity by the bulk electrical conductivity which will therefore allow the effects of changing micro-structure to be evaluated irrespective of material used.

Figure 7 shows the ratio of effective to bulk electrical conductivity for all of the structures tested within this study. All ordered microstructures have a higher TP and IP conductivity than the GDL TP however only the isotropic cubic lattice performs better than both IP and TP of the GDL. This is because the Hex Lattice has a greater TP conductivity than all structures and therefore, due to its anisotropy must lose effective conductivity in the IP direction. The effect of the differences in conductivity between the microstructure can be attributed to the electron tortuosity and effective porosity as shown by the analytical analysis of fiber structures in Equation 20. Consequently the findings from the simulation show that ordered microstructures are able to achieve greater electrical conductivity than unstructured systems such as a GDL. This increase in electrical conductivity will allow for a GDL that is thinner and lower in resistance.

To further analyze the mechanisms of the electron transport in terms of the physical quantities that were derived, the fibrous GDL was reconstructed for 5 cases with varied anisotropy $(\beta=1,5,10,20,40)$ at 3 different porosity levels $(\epsilon=0.6,0.7,0.8)$ which allowed the structures to have variation in the angle between the $\mathrm{x} / \mathrm{y}$ and $\mathrm{z}$ directions. The electrical conductivity of these structures was simulated and the electron tortuosity was extracted from the streamlines using the definition in Equation 18. The results in Figure 8 show that at a constant porosity, both the IP and TP directions for conductivity roughly lie on the same curve which is dependent on the electron tortuosity. This implies that the effective electrical conductivity is a function of both porosity and electron tortuosity. In order to improve predictive capabilities, the simulation data can be transformed which allows for separation of data points resulting from the difference in porosity.

The angle between each line in Figure 9 is a function of porosity and therefore to collapse the data onto a line. The gradient of each line changes with porosity, meaning the equation relating the root of conductivity $\sigma$ is in the form

$$
\sqrt{\sigma}=\frac{C}{\tau_{e}}
$$

The constant $C$ is a function of porosity, by determining the difference in the gradient of the lines in Figure 9, $C$ was found, resulting in the 


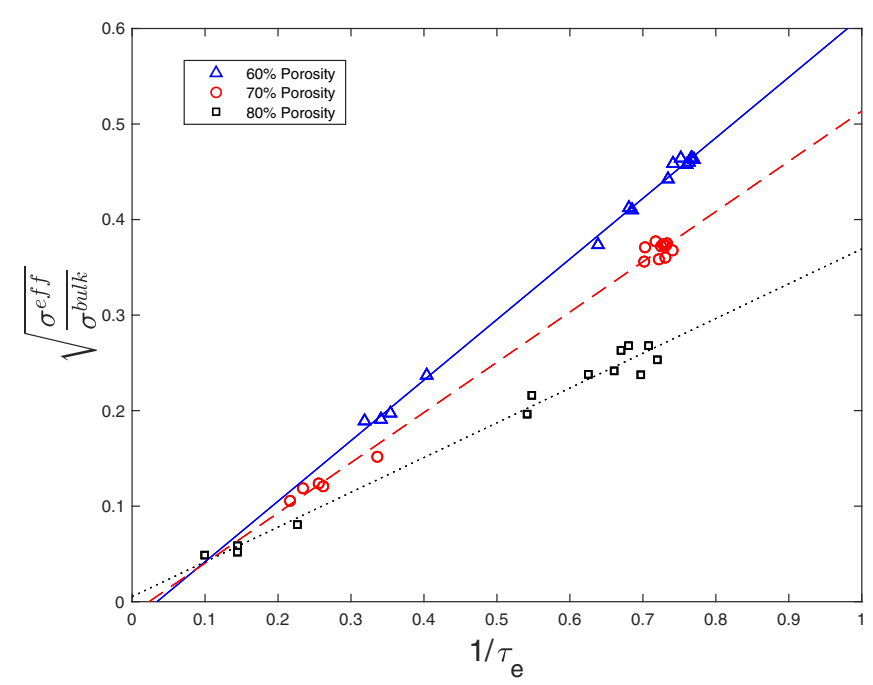

Figure 9. Transformation through square root of the dimensionless electrical conductivity $\sqrt{\sigma^{e f f} / \sigma^{b u l k}}$ from Figure 8 against the reciprocal of electron tortuosity, allowing the results to lie on linear functions determined by porosity of the microstructures. The blue solid line, red dashed line and black dotted line represents $0.6,0.7$ and 0.8 porosity respectively.

final equation that relates electrical conductivity of the microstruture to the porosity and the electron tortuosity.

$$
\sqrt{\sigma}=\frac{-0.73 \epsilon+1.07}{\tau_{e}}
$$

This equation was developed based on fibrous porous media, and is similar to Equation 20 developed analytically for the cubic lattice structures. Equation 23 is able to predict the effective electrical conductivity reasonably well only based upon the microstructural properties of porosity and electron tortuosity. Porosity is easily extractable from experimental data however, methods to quickly characterize electron tortuosity from imaging should be developed if this equation can be used effectively.

Comparison between the predictive capability of Equation 23 with results shown in Figure 10 to the analytical developed Equation 20 in Figure 11 for all structures allows for further validation and analysis of both equations. Figure 10 shows the high predictive capability between simulation and theoretical work for both GDL and lattice

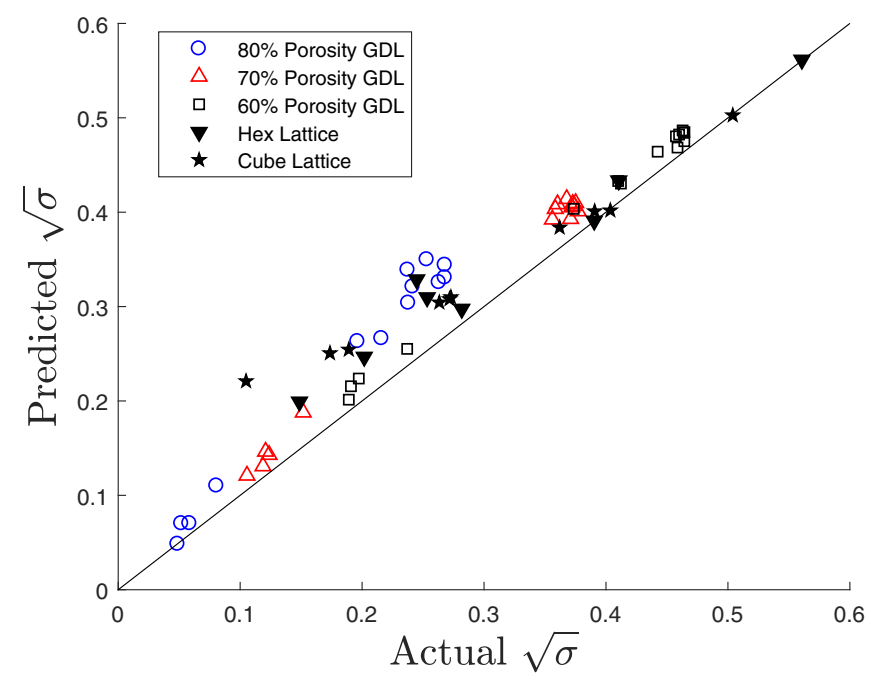

Figure 10. Predictive capability of Equation 23 for determining the square root of the dimensionless effective electrical conductivity $\sqrt{\sigma}$ of GDL and lattice structures compared to simulation data with a $R^{2}=0.895$.

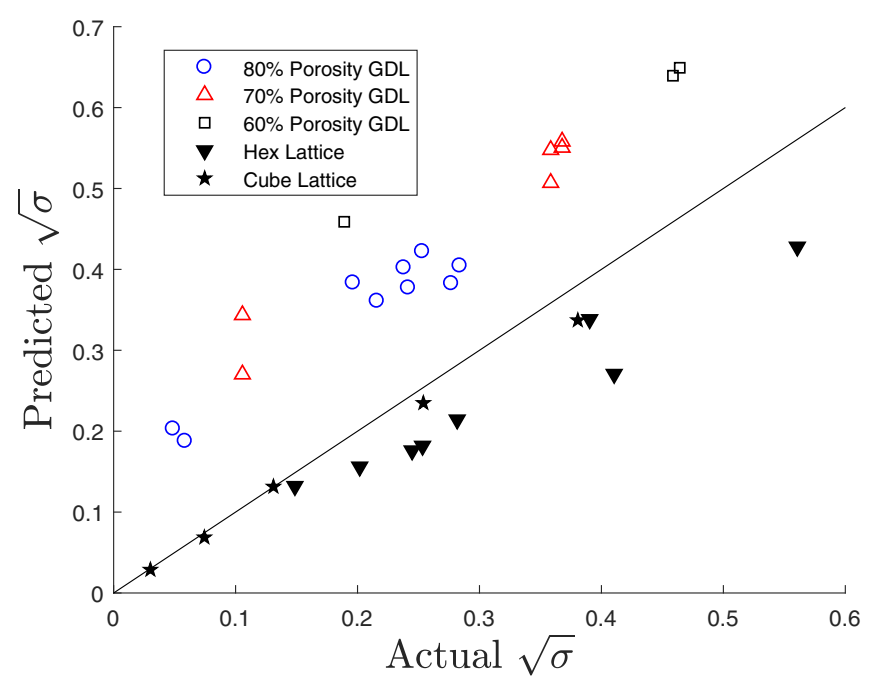

Figure 11. Predictive capabilities of Equation 20 at determining the square root of the dimensionless ratio of effective to bulk electrical conductivity $\sigma$ for the different microstructures tested. The analytical Equation 20 was developed for the cubic lattice model which is shown by its greater predictive capability $R^{2}=0.997$ for the cubic lattice alone but very low $R^{2}=0.25$ for all structures combined.

structures of Equation 23 with a $R^{2}$ of 0.895 . Alternatively Figure 11 shows the predictive capability of Equation 20 which is the analytical equation developed for a cubic lattice and therefore has no empirical constants which is more accurate for the cubic lattice than the semiempirical Equation 23, but this is only valid for cubic lattice structures. Consequently, resulting from the difference between the predictive capability between Figure 10 and Figure 11, it seems only applicable to use Equation 23, given that the microstructural properties of electron tortuosity and porosity or effective porosity are known. The use of either of these equations is useful for quick analysis into determining if a structure will be more conductive than a current GDL and this can even be used for other applications where the porous solid micro-structure is conductive.

Furthermore, it is clear from the overprediction of GDL structure electrical conductivity in Figure 11 that the 2D cross sectional effective porosity, is not the real area that the electrons flow through. Therefore, Equation 20 over predicts the electrical conductivity for unstructured GDL, since electrons will follow a stream-tube, so their effective area to flow through would be different from a 2D cross section. Regardless, the cubic lattice structures can be predicted accurately with this equation with $R^{2}=0.997$.

Application of these equations requires the electron tortuosity which should be defined by the geometric tortuosity, however it appears from the simulation and the parameterisation of the analytical equation that at the connection between fibers, there is an induced tortuosity. This could be related to the construction of the cubic lattice; at higher porosities, the difference between the minimum and maximum area for electron flow is larger.

The electron tortuosity found by parameterisation of Equation 20 is similar to the the values found from the computational simulations. For a bundle of tubes that do not intersect, the tortuosity is 1, however resulting from the connections between fibers the straight tubes within a cubic lattice receive an induced electron tortuosity, this effect can be seen by the streamlines of electron transport in the cubic structures as Figure 12 shows.

Permeability.-Simulation of single phase flow through the void space generated by the different micro-structures allowed for the determination of the permeability tensor. The validation of the model is based upon the comparison of the GDL reconstructions with experimental data in literature ${ }^{27}$ shown in Figure 13 . Although permeability 


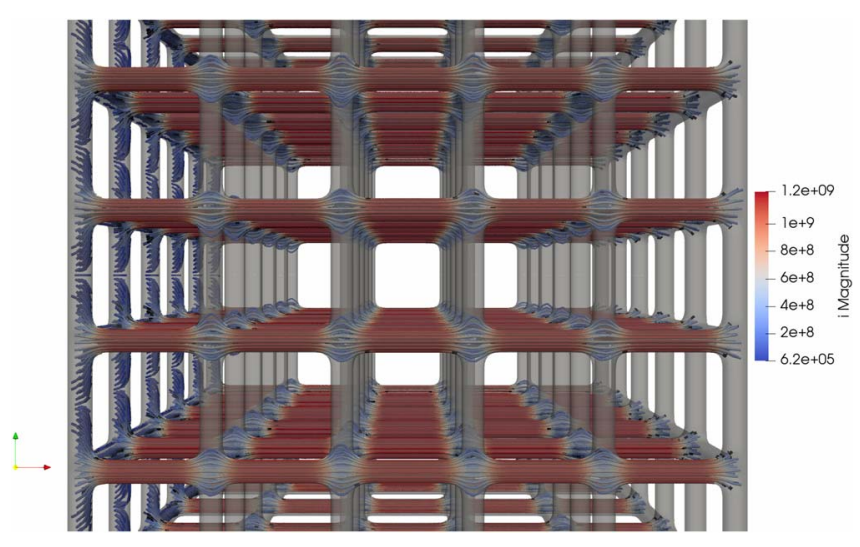

Figure 12. Visualisation of electron flow through a cubic lattice with a fiber diameter of $8 \mu \mathrm{m}$ and fiber length of $40 \mu \mathrm{m}$. At the network interconnections, the streamlines deviate from the path, inducing tortuosity.

does not define its ability for management of water, having an increased permeability will increase the potential for the amount of air or water that can pass through the space.

The difference between the TP simulation and experimental results shown in Figure 13 are likely due to the the realistic representation not being able to be realized in a random stochastic process. Note that the exact 3D structure of the GDL in the referenced studies were not available and a significant difference between the simulated and measured permeability was expected, Also we don't have sufficient information of the experimental setup and procedure. For example GDL layers were stacked together, developing large spaces at the interface between layers. ${ }^{27}$ Figure 13 allowed confidence in the solver and a benchmark to which regular ordered structures can be tested against.

As a comparison, by fixing the fiber diameter, the ordered structure dimensions can be manipulated to create variation in porosity. This allows comparison with the GDL because of the same volume domain used and same fiber diameter, meaning that the only the topology of the system has changed. Results of permeability simulations in Figure 14 show that both ordered micro-structures have higher TP permeability than the GDL. However, due to its anisotropy, the hex lattice fails to have a larger IP permeability than the GDL. The cubic lattice has

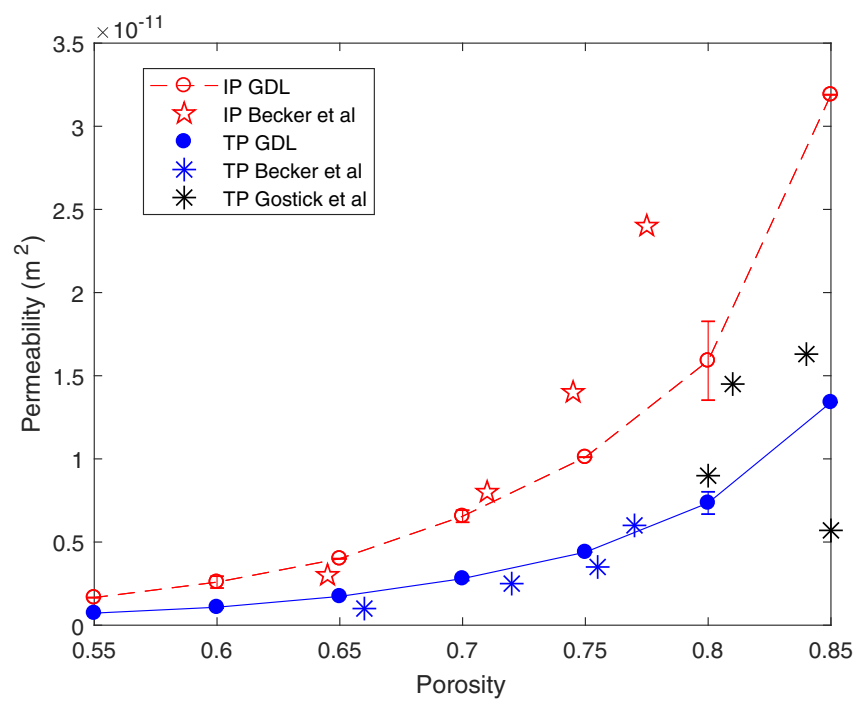

Figure 13. Comparison of the simulated permeability for GDL reconstructions at different porosity levels with experimental data from literature. The hollow and solid points represent the IP and TP directions respectively. Error bars show the standard deviation between the domain sizes tested.

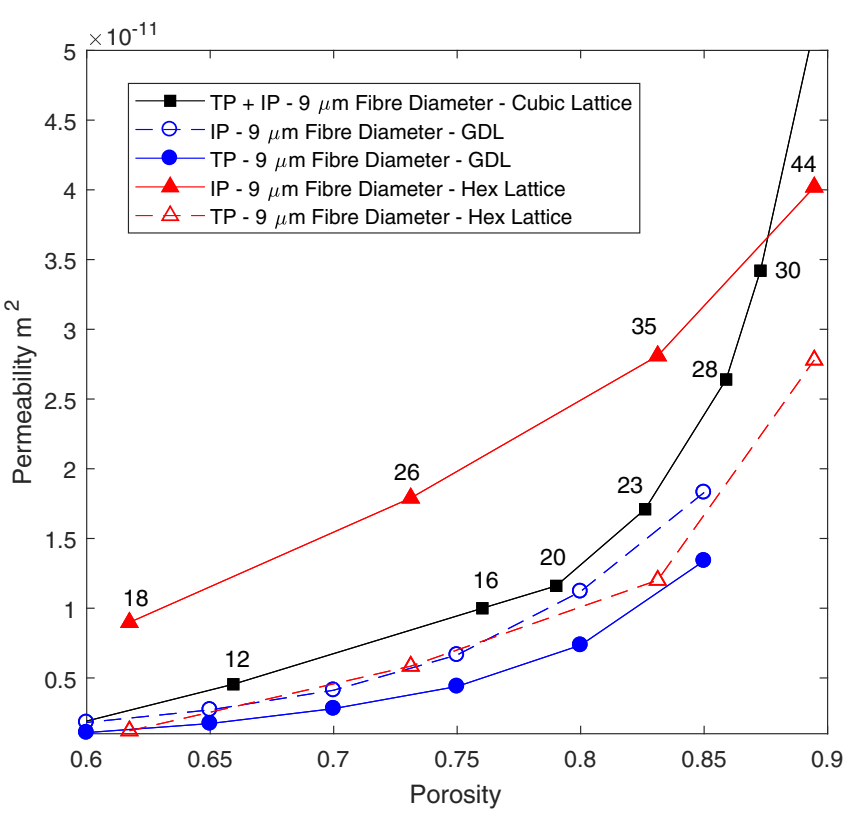

Figure 14. Comparison of permeability of GDL structures and ordered structures, with a fiber diameter of $9 \mu \mathrm{m}$. The numbers next to each point represents the inscribed diameter $(\mu \mathrm{m})$ of the void space pores for the cubic lattice (black square) and hexagonal lattice (red triangle).

higher permeability in both TP and IP directions compared to the GDL because it is isotropic. The difference in permeability between the cubic and hex lattice is due to the anisotropy; the hex lattice can achieve greater a inscribed diameter at the same porosity as shown by the numbers on Figure 14.

The lattice structures are highly customisable, meaning that the permeability can be increased by simply scaling the system. The optimization of permeability is possible through microstructural design however, its effects on fuel cell performance cannot be established through this transport property alone; the two-phase system will determine how effective the structure is. Nevertheless at a given domain size, the optimization between permeability and effective electrical conductivity can be established.

Optimisation of transport properties for a cubic lattice.-As shown in Figure 7 and Figure 14 there is a point where both transport properties are maximized for any structure. However, for an optimized performance both electrical conductance and hydraulic permeability are important. Currently, we do not have any weighting for these curves and to validate the optimum porosity for a given GDL structure, further full system modelling or experimental work are needed. Assuming equal weighting, the optimum porosity of a structure will be at 0.85 , as shown in Figure 15.

Using Figure 15a cubic lattice structure porosity can be chosen so that the transport properties are greater than that of conventional GDL. In the case of the simulations performed in this study, the GDL with highest permeability and lowest electrical conductivity obtained was $3.2 \times 10^{-11} \mathrm{~m}^{2}$ and $130 \mathrm{~S} \mathrm{~m}^{-1}$ respectively, meaning that any porosity cubic lattice greater than around 0.85 porosity will have a larger permeability than a GDL, including a larger conductivity.

\section{Discussion and Conclusions}

Creating and utilizing an improved GDL will allow for a more homogeneous transfer of both electrons and fluids meaning that there will be more potential for phases to flow. This should reduce the resistances associated with the solid phase, meaning thinner GDL's could be utilized, whilst further improving the current density able to be achieved 


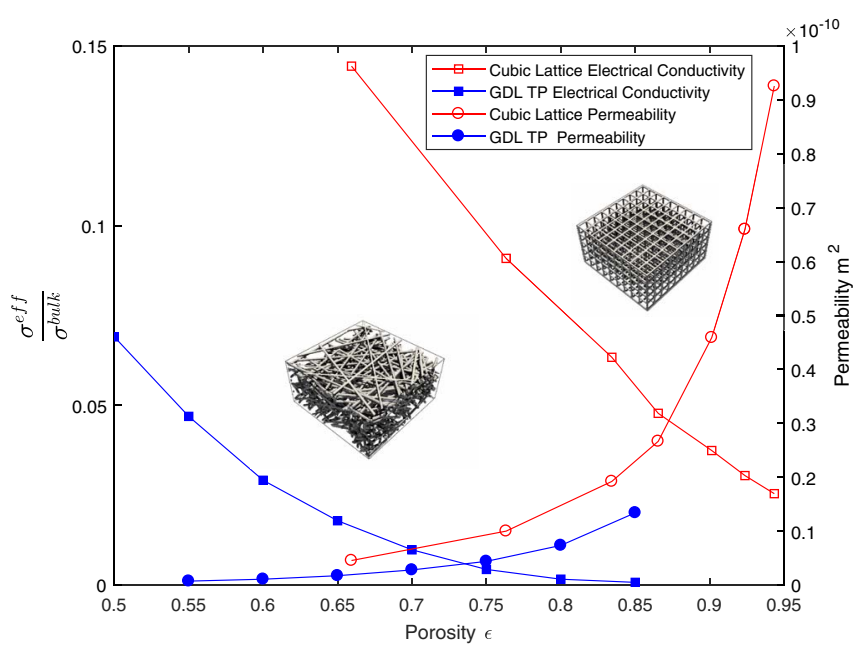

Figure 15. Variation in the ratio of effective electrical conductivity to bulk conductivity and permeability of a cubic lattice of dimensions shown above the red points and a fiber diameter of $9 \mu \mathrm{m}$, optimization can be performed by selecting the desired transport properties for the Cubic Lattice. Carbon paper reconstructed properties can be found in the blue points.

at the same voltage due to improved transport of air and water in the layer.

By using 3D printed micro-structures there will be improved continuity between solid phases; resulting in lower contact resistances between fibers. Additionally, due to the periodic nature of such structures, there will be a more uniform current to the catalyst layer, whilst also providing better mechanical support leading to less deformation of the GDL, CL and membrane. This is because lattice structures have a high strength to density ratio and it will be less likely that there will be breakages between fibers if the structural integrity is taken into account. To summarize the potential improvements to be made by implementation of a 3D printed morphology controlled micro-structure are:

- Improved effective electrical conductivity

- Improved two-phase transport properties

- Improved continuity within each phase; there is no breakages in the solid or void spaces

- Uniform current to catalyst layer

- Reduced contact resistances due to larger contact between bipolar plate and CL

- Reduced cost and size

- Improved mechanical integrity

In fuel cell operation, reaction of oxygen at the catalyst layer results in the production of water; resulting in two-phase flow. To fully understand the effect of using a structured morphology approach on fuel cell performance, simulation studies must be conducted. This work is underway and will assess the performance of a benchmark GDL to a $3 \mathrm{D}$ printed layer. In this study we aimed assessed the influence of microstructrual properties on the basic transport properties that govern the performance of a GDL in a fuel cell.

The effective electrical conductivity and permeability of any microstructure of both hex lattice and cubic lattice GDL, were compared against unstructured GDL. The addition of a controlled morphology GDL will allow for a greater fuel cell performance, allowing for higher current densities being able to be reached at the same voltage used in a conventional GDL system. The GDL using a cubic lattice design, could be tailored to the current as at high current density, fluid transport limitations are more important and at low current, high electrical conductivity is important.

Moreover, if the fibers of the 3D printed lattices can be manufactured from the same material, using the appropriate carbonisation method and accounting for shrinkage, it is likely that the other trans- port properties will improve and therefore the fuel cell performance will improve.

Although the isotropic arrangement gives the most ideal and nontortuous pathway for electron transport and diffusion, the surface wettability and pore throat size arrangement will effect the two-phase flow properties of ordered micro-structures. If these are to be considered, it is likely that an arrangement of fibers with larger pore throat sizes in the through-plane direction compared to the in-plane direction in an cubic lattice like arrangement proposed in Figure 3 will result in the least interacting non-tortuous pathway.

\section{Acknowledgments}

The authors would like to acknowledge the UK Engineering and Physical Sciences Research Council (EPSRC) for funding this PhD iCASE award studentship for Daniel Niblett. Furthermore, we would like to thank Philippe Paliard at Microlight 3D for manufacturing a prototype sample shown in Figure 3.

\section{ORCID}

Daniel Niblett (iD https://orcid.org/0000-0003-3532-7532

Vahid Niasar (10 https://orcid.org/0000-0002-9472-555X

\section{References}

1. M. E. Hannach and E. Kjeang, "Stochastic microstructural modeling of pefc gas diffusion media," Journal of Electrochemical Society, 161(9), F951 (2014).

2. B. Tjaden, S. J. Cooper, D. J. Brett, D. Kramer, and P. R. Shearing, "On the origin and application of the bruggeman correlation for analysing transport phenomena in electrochemical systems," Current Opinion in Chemical Engineering (2016).

3. N. Zamel, X. Li, and J. Shen, "Numerical estimation of the effective electrical conductivity in carbon paper diffusion media," Applied Energy, 93, 39 (2012).

4. A. Forner-Cuenca, J. Biesdorf, L. Gubler, P. M. Kristiansen, T. J. Schmidt, and P. Boillat, "Engineered water highways in fuel cells: Radiation grafting of gas diffusion layers," Advanced Materials, 27, 6317 (2015).

5. Y. Daicho, T. Murakami, T. Hagiwara, and S. Maruo, "Formation of three-dimensional carbon microstructures via two-photon microfabrication and microtransfer molding," Optical Materials Express, 3(6), 875 (2013).

6. M. Shojaeefard, G. Molaeimanesh, M. Nazemian, and M. Moqaddari, "A review on microstructure reconstruction of pem fuel cells porous electrodes for pore scale simulation," International Journal of Hydrogen Energy, 41, 20276 (2016).

7. P. A. Garcia-Salaberri, I. V. Zenyuk, A. D. Shum, G. Hwang, M. Vera, A. Z. Weber, and J. T. Gostick, "Analysis of representative elementary volume and through-plane regional characteristics of carbon-fiber papers: diffusivityl, permeability and electri$\mathrm{cal} /$ thermal conductivity," International Journal of Heat and Mass Transfer, 127, 687 (2018).

8. A. Sharma and A. K. Nagar, "A mathematical model for performance of proton exchange membrane fuel cell as a nonlinear voltage processes," International Journal of Advanced Research in Computer Science, 8(1), 27 (2017).

9. L. Li, S. Bei, Q. Xu, K. Zheng, and Y. Zheng, "Role of electrical resistance and geometry of porous electrodes in the performance of microfluidic fuel cells," International Journal of Energy Research (2017).

10. M. Ismail, K. Hughes, D. Ingham, L. Ma, and M. Pourkashanian, "Effects of anisotropic permeability and electrical conductivity of gas diffusion layers on the performance of proton exchange membrane fuel cells," Applied Energy, 95, 50 (2012).

11. T.-F. Cao, H. Lin, L. Chen, Y.-L. He, and W.-Q. Tao, "Numerical investigation of the coupled water and thermal management in pem fuel cell," Applied Energy, 112 (2013).

12. S. Li, J. Yuan, M. Andersson, G. Xie, and B. Sunden, "Influence of anisotropic gas diffusion layers on transport phenomena in a proton exchange membrane fuel cell," International Journal of Energy Research, 41, 2034 (2017).

13. L. Li, W. Fan, J. Xuan, and M. K. Leung, "Dimensionless parametric sensitivity analysis of microfluidic fuel cell with flow-through porous electrodes," Electrochimica Acta, 187, 636 (2016).

14. G. Zhang, L. Fan, J. Sun, and K. Jiao, "A $3 \mathrm{~d}$ model of pemfc considering detailed multiphase flow and anisotropic transport properties," International Journal of Heat and Mass Transfer, 115, 714 (2017).

15. J. T. Gostick, M. W. Fowler, M. D. Pritzker, M. A. Ioannidis, and L. M. Behra, "Inplane and through-plane gas permeability of carbon fiber electrode backing layers," Journal of Power Sources, 162, 228 (2006).

16. O. J. A. Schueller, S. Brittain, and G. M. Whitesides, "Fabrication of glassy carbon microstructures by pyrolysis of microfabricated polymeric precursors," Advanced Materials, 9(7), 477 (1996).

17. J. Mo, R. R. Dehoff, W. H. Peter, T. J. Toops, J. B. Green Jr., and F.-Y. Zhang, "Additive manufacturing of liquid/gas diffusion layers for low-cost and high-efficiency hydrogen production," International Journal of Hydrogen Energy, 41, 3128 (2016). 
18. A. Jayakumar, S. Singamneni, M. Ramos, A. M. Al-Jumaily, and S. S. Pethaiah, "Manufacturing the gas diffusion layer for pem fuel cell using a novel $3 \mathrm{~d}$ printing technique and critical assessment of the challenges encountered," Materials, 10 (2017).

19. S. Torquato, S. Hyun, and A. Donev, "Optimal design of manufacturable threedimensional composites with multifunctional characteristics," Journal of Applied Physics, 94(9) (2003).

20. Y. Jung and S. Torquato, "Fluid permeabilities of triply periodic minimal surfaces," Physical Review E, 72, 1 (2005).

21. J. K. Guest and J. H. P. Vost, "Design of maximum permeability material structures," Computer Methods in Applied Mechanics and Engineering, 196, 1006 (2007).

22. Pablo A. Garcia-Salaberri and I. I. Marcos Vera, "Modeling of the anode of a liquidfeed dmfc: Inhomogeneous compression effects and two-phase transport phenomena," Journal of Power Sources, 246, 239 (2014).

23. V. P. Schulz, J. Becker, A. Wiegmann, P. P. Mukherjee, and C.-Y. Wang, "Modeling of two-phase behavior in the gas diffusion medium of pefcs via full morphology approach," Journal of The Electrochemical Society, 154(4), B419 (2007).
24. M. M. Daino and S. G. Kandlikar, "3d phase-differentiated gdl microstructure generation with binder and ptfe distributions," International Journal of Hydrogen Energy, 37, 5189 (2012)

25. D Fadzillah, M. Rosli, M. Talib, S Kamarudin, and W. Daud, "Review on microstructure modelling of a gas diffusion layer for proton exchange membrane fuel cells," Renewable and Sustainable Energy Reviews, 77, 1001 (2017).

26. A. El-kharouf, T. J. Mason, D. J. Brett, and B. G. Pollet, "Ex-situ characterisation of gas diffusion layers for proton exchange membrane fuel cells," Journal of Power Sources, 218, 393 (2012).

27. J. Becker, R. Fluckiger, M. Reum, F. N. Buchi, F. Marone, and M. Stampanonic, "Determination of material properties of gas diffusion layers: Experiments and simulations using phase contrast tomographic microscopy," Journal of The Electrochemical Society, 156(10), B1175 (2009).

28. R. Guibert, M. Nazarova, P. Horgue, G. Hamon, P. Creux, and G. Debenest, "Computational permeability determination from pore-scale imaging: Sample size, mesh and method sensitivities," Transport in Porous Media (2015). 